\title{
BMJ Open Antenatal antiarrhythmic treatment for fetal tachyarrhythmias: a study protocol for a prospective multicentre trial
}

Takekazu Miyoshi, ${ }^{1}$ Yasuki Maeno, ${ }^{2}$ Haruhiko Sago, ${ }^{3}$ Noboru Inamura, ${ }^{4}$ Satoshi Yasukochi, ${ }^{5}$ Motoyoshi Kawataki, ${ }^{6}$ Hitoshi Horigome, ${ }^{7}$ Hitoshi Yoda, ${ }^{8}$ Mio Taketazu, ${ }^{9}$ Makio Shozu, ${ }^{10}$ Masaki Nii, ${ }^{11}$ Akiko Hagiwara, ${ }^{12}$ Hitoshi Kato, ${ }^{13}$ Wataru Shimizu, ${ }^{14}$ Isao Shiraishi, ${ }^{15}$ Heima Sakaguchi, ${ }^{15}$ Keiko Ueda, ${ }^{1}$ Shinji Katsuragi, ${ }^{1}$ Tomoaki Ikeda, ${ }^{16}$ Haruko Yamamoto, ${ }^{17}$ Toshimitsu Hamasaki, ${ }^{18}$ Japan Fetal Arrhythmia Group

To cite: Miyoshi T, Maeno Y, Sago $\mathrm{H}$, et al. Antenatal antiarrhythmic treatment for fetal tachyarrhythmias: a study protocol for a prospective multicentre trial. BMJ Open 2017;7:e016597. doi:10.1136/ bmjopen-2017-016597

- Prepublication history for this paper is available online To view these files please visit the journal online (http://dx.doi org/10.1136/bmjopen-2017016597).

Received 25 February 2017 Revised 24 May 2017 Accepted 25 July 2017

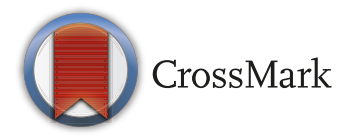

For numbered affiliations see end of article.

Correspondence to

Dr Yasuki Maeno;

yasukim@med.kurume-u.ac.jp

\section{ABSTRACT}

Introduction Several retrospective or single-centre studies demonstrated the efficacy of transplacental treatment of fetal tachyarrhythmias. Our retrospective nationwide survey showed that the fetal therapy will be successful at an overall rate of $90 \%$. For fetuses with hydrops, the treatment success rate will be $80 \%$. However, standard protocol has not been established. The objective of this study is to evaluate the efficacy and safety of the protocol-defined transplacental treatment of fetal tachyarrhythmias. Participant recruitment began in October 2010.

Methods and analysis The current study is a multicentre, single-arm interventional study. A total of 50 fetuses will be enrolled from 15 Japanese institutions. The protocol-defined transplacental treatment is performed for singletons with sustained fetal tachyarrhythmia $\geq 180 \mathrm{bpm}$, with a diagnosis of supraventricular tachycardia or atrial flutter. Digoxin, sotalol, flecainide or a combination is used for transplacental treatment. The primary endpoint is disappearance of fetal tachyarrhythmias. The secondary endpoints are fetal death related to tachyarrhythmia, proportion of preterm birth, rate of caesarean section attributable to fetal arrhythmia, improvement in fetal hydrops, neonatal arrhythmia, neonatal central nervous system disorders and neonatal survival. Maternal, fetal and neonatal adverse events are evaluated at 1 month after birth. Growth and development are also evaluated at 18 and 36 months of corrected age.

Ethics and dissemination The Institutional Review Board of the National Cerebral and Cardiovascular Center of Japan has approved this study. Our findings will be widely disseminated through conference presentations and peerreviewed publications.

Trial registration number UMIN Clinical Trials Registry UMIN000004270.

\section{INTRODUCTION}

Fetal tachyarrhythmia develops in less than $0.1 \%$ of pregnancies. The most common forms of tachyarrhythmia are supraventricular tachycardia (SVT) and atrial flutter

\section{Strengths and limitations of this study}

- This is the first multicentre prospective study to confirm the efficacy and safety of the protocoldefined transplacental treatment of fetal tachyarrhythmias.

- Fetal treatment protocol was carefully constructed depending on the ratio of atrioventricular and ventriculoatrial intervals by using fetal Doppler echocardiography.

- A possible limitation is related to the single-arm interventional study. The results of our retrospective survey and historical control data from the literature are referenced in designing this clinical study.

- Results will be limited to the efficacy of digoxin, sotalol and flecainide for fetal supraventricular tachycardia and atrial flutter.

(AFL). Fetal arrhythmia with 1:1 atrioventricular (AV) conduction at rates of 180-200 bpm might include some cases of sinus tachycardia such as infection, anaemia, fetal distress, medication use, trauma or hyperthyroidism in the mother. ${ }^{12}$ Fetal tachyarrhythmia results in a wide spectrum of outcomes, including spontaneous remission, heart failure, fetal hydrops and fetal death. In fetuses, severe or long-lasting tachyarrhythmia often leads to heart failure and fetal hydrops. ${ }^{3}{ }^{4}$ Fetal hydrops has been reported in $30 \%-40 \%$ of fetuses with SVT and in $7 \%-43 \%$ of those with AFL. ${ }^{56}$ High incidences of fetal hydrops are associated with tachycardia lasting for 12 or more hours. For fetuses with fetal hydrops, elective preterm delivery and other life-saving interventions are often attempted. Yet, studies have reported a $35 \%$ perinatal mortality rate with fetal hydrops. Since few studies have reported cases of fetal hydrops without preterm intervention, we cannot 
accurately predict the natural prognosis of the disease. Available data suggest that the risk of fetal death will be elevated if tachyarrhythmia is left untreated in fetuses with fetal hydrops.

It has long been known that administration of antiarrhythmic drugs in a pregnant woman with fetal tachyarrhythmia has improvements in sinus rhythm of her fetus, and efforts have been made for transplacental treatment. ${ }^{7-10}$ Those interventions typically have reported improvements in sinus rhythm in $\geq 80 \%$ of fetuses without fetal hydrops. In addition, many studies have reported the therapeutic effectiveness in fetuses with fetal hydrops. Reported successes in transplacentally treating fetal tachyarrhythmia, combined with the relative ease of the procedure, have increased the popularity of this approach in Japan and many other countries. Accumulated evidence of transplacental pharmacotherapy for fetal tachyarrhythmia has prompted obstetricians and paediatricians to establish a standard protocol for this approach. Although a lot of institutions have reported standardised protocols, majority of reports are single-centre retrospective studies. These reports have limitation in design, including institution biases and differences in the inclusion or exclusion criteria. In addition, many of the retrospective studies are difficult to collect maternal, fetal and neonatal adverse events. To the best of our knowledge, there are only two prospective studies. ${ }^{10}{ }^{11}$ However, these are single-centre and relatively small sample size which is less than 20 cases. Therefore, we are now performing the larger multicentre prospective study to confirm the efficacy and safety of the protocol-defined transplacental treatment of fetal tachyarrhythmias.

The choice of first and second-line antiarrhythmic therapy and criteria for decisions about management after initial treatment failure are controversial. In many centres, digoxin, administered maternally either orally or intravenously, is used as first-choice therapy because of its relatively safe profile, its long history of use during pregnancy and the familiarity with its use. Sotalol, flecainide and amiodarone have all been used as second-choice therapy. ${ }^{12-14}$ However, amiodarone has a more significant toxicity profile for the mother and fetus. ${ }^{14}$ Therefore, we selected digoxin as the first-choice drug, sotalol as the second-choice drug and flecainide as the third-choice drug in this study protocol. Digoxin has been explained by incomplete passage of the drug across the placenta, whereas sotalol and flecainide continue to cross the placenta readily even if there is fetal hydrops and should be preferred for patients in heart failure. Combination therapy with sotalol or flecainide is selected in hydropic fetuses.

The precise identification of the mechanism underlying a tachyarrhythmia is important since the pharmacological approach can be quite different. It is important to give the most efficient drug at the lowest possible dose and to avoid as much as possible blind trials of multiple drugs that increase the risk of maternal and fetal morbidity. To classify tachyarrhythmia, the efficacy of AV and ventriculoatrial (VA) intervals based on Doppler echocardiography is reported. ${ }^{11516}$ Short VA SVT is the typical pattern in re-entry tachycardia. All drugs can be expected to be effective against the majority of re-entrant tachycardia found in utero. In this study protocol, digoxin is used as first-choice therapy because of the vast experience gained with this agent in prenatal antiarrhythmic treatment. On the other hand, long VA SVT is rare tachycardia, suggesting atrial ectopic tachycardia or permanent junctional reciprocating tachycardia. Since digoxin was reported to be ineffective in the long VA SVT, ${ }^{17}$ sotalol is used as first-choice therapy in this study protocol.

The primary goal of this study is to develop clinical guidelines for safe and effective fetal tachyarrhythmia treatment. This required us to evaluate the current status of treatments and results in Japan, and demonstrate the safety and effectiveness of fetal therapy in a rigorous clinical trial. Therefore, we planned a multicentre prospective study to confirm the efficacy and safety of the protocol-defined transplacental treatment of fetal tachyarrhythmias.

\section{METHODS}

\section{Study design}

This trial is a multicentre, single-arm interventional study. Figure 1 shows the study design in detail.

\section{Study period}

The planned study period is from the ethics approval to March 2021. The patient registration period will last until December 2017. The neonatal evaluation period will continue until 1 month after the last delivery. Data collected by the end of the neonatal evaluation period will be subjected to statistical analysis. The children's growth and development will be followed up for 3 years after birth. Growth and development assessments will be defined in more details according to a separate protocol.

\section{Sample size}

Sample size in this study is 50 fetuses. The target disorders of this clinical study are very rare, with a rate of 1 out of

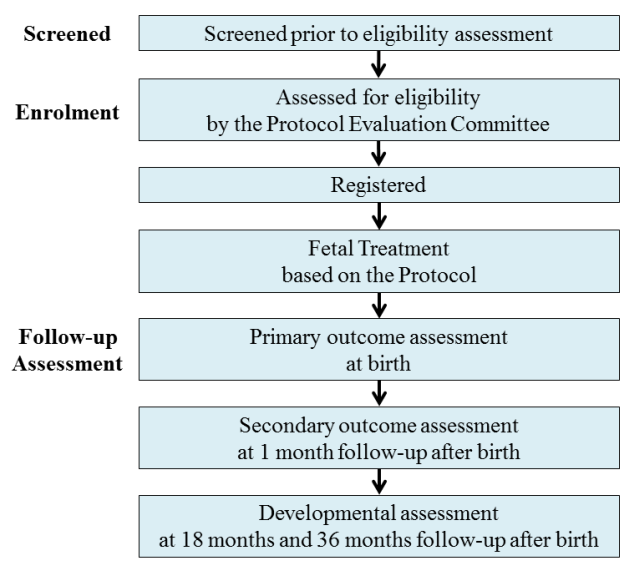

Figure 1 Summary of the study design. 
1000 pregnancies. At the National Cerebral and Cardiovascular Center, six cases were treated in the period from 2004 to 2006 . We expect that a total of 50 cases will be treated over a 5-year period at the participating study sites. A sample size of 50 produces a two-sided, Clopper-Pearson exact $95 \%$ CI with a width equal to 18.5 when the sample proportion is assumed $90 \%$.

\section{Endpoints}

The primary endpoint of this study is disappearance of fetal tachyarrhythmia. The disappearance or dissipation of fetal tachyarrhythmia must fulfil either or both of the following conditions: normal sinus rhythm or mean heart rate $<180 \mathrm{bpm}$. The secondary endpoints are fetal death definitely or probably related to tachyarrhythmia, proportion of preterm birth, rate of caesarean section attributable to fetal arrhythmia, improvement in heart rate and oedema after the fetal therapy, neonatal arrhythmia rate, neonatal central nervous system disorders and survival at 1 month of age.

The safety endpoints are maternal, fetal and neonatal adverse events. Maternal adverse events are arrhythmia, ECG abnormalities, hepatic or renal dysfunction, dizziness, nausea, vomiting and worsening or onset of obstetric complications, such as preterm delivery, gestational hypertension, haemorrhage and gestational diabetes mellitus. ECG abnormalities are defined as PR prolongation, AV block, QT prolongation of more than $500 \mathrm{~ms}$, and so on. Fetal adverse events are bradycardia, organ dysfunction and a new onset of arrhythmia. Neonatal adverse events are arrhythmia, ECG abnormalities, hepatic or renal dysfunction, respiratory disorders, sustained cyanosis and jaundice.

\section{Patient selection}

Inclusion criteria are as follows: (1) sustained fetal tachyarrhythmia $\geq 180 \mathrm{bpm}$, with a diagnosis of SVT or AFL. Fetal tachyarrhythmia is defined as sustained when present $\geq 50 \%$ during a $40 \mathrm{~min}$ fetal heart rate monitoring or 30 min echocardiography session; (2) 22 to $<37$ weeks of gestation; (3) singleton pregnancy; (4) the parents' written consent to study participation.

Exclusion criteria are as follows: (1) the mother has mental or psychiatric conditions that preclude safe study participation; (2) the mother is anticipated to be contraindicated to the antiarrhythmic medications; (3) the fetus is diagnosed to have serious and life-threatening malformations; (4) the fetus is diagnosed with multifocal atrial tachycardia or chaotic atrial tachycardia; (5) the mother or fetus is not eligible, in the investigator's judgement, for this clinical study for reasons other than those above.

\section{Subjects and diagnostic methods}

This clinical trial includes fetuses with SVT or AFL. These are diagnosed by using fetal echocardiography. SVT is defined as a tachyarrhythmia of supraventricular origin, characterised by 1:1 AV conduction. Depending on the ratio of $\mathrm{AV}$ and VA intervals, SVTs with a VA interval shorter than the AV (ie, VA/AV ratio $<1$ ) are designated as 'short VA,' and SVTs with a VA interval longer than the AV (ie, VA/AV ratio >1) are designated as 'long VA.' The VA interval relates to the time between the onset of a ventricular $(\mathrm{V})$ waveform to the onset of an atrial (A) waveform, while the AV interval relates to the time between the onset of an A waveform to the onset of a V waveform. The classification of short and long VA types of fetal tachyarrhythmia will primarily be made based on Doppler echocardiography, with supplementary assistance of M-mode echocardiography. VA intervals will be determined according to one of the following methods that evaluate: (1) waveforms of the ascending aorta and superior vena cava; (2) waveforms of the left ventricular inflow and outflow; (3) pulmonary artery and vein waveforms. If the SVT cannot be classified as either long or short VA type for certain reasons, it will be categorised as the short VA type for the sake of initiating study treatment. Fetuses diagnosed with multifocal atrial tachycardia or chaotic atrial tachycardia will be excluded from this study. AFL is defined as a tachyarrhythmia of supraventricular origin, characterised by 2:1 or greater AV conduction rate due to AV blocking. To ensure the diagnostic accuracy, the investigator will record the fetal ultrasound scans (photo or video) at the time of diagnosis, and the scans will be reviewed by the Protocol Evaluation Committee.

\section{Registration}

Patients who satisfy all inclusion criteria and do not meet any of the exclusion criteria will be eligible for inclusion in the study. Individual study sites will be responsible for guiding potential participants through the informed consent process, including those patients who have been referred to them for treatment purposes. The investigator will enter an eligible patient's information into the Eligibility Confirmation Form and send it by fax to the Secretariat in the National Cerebral and Cardiovascular Center. The Secretariat will check the contents of the form before registering the patient. Enrolled participants will receive fetal therapy within 7 days of registration.

The Protocol Evaluation Committee is an organisation independent of the execution of this study. This committee will review the accuracy of the fetal diagnosis retrospectively to ensure the overall quality of this clinical study. As a basic rule, the opinion of this committee will not impact the fetal therapy or other treatment-related aspects of this clinical study. The investigator will mail the ultrasound video of each registered mother recorded at diagnosis to the Protocol Evaluation Committee. At the completion of this study, the Protocol Evaluation Committee will review the legitimacy of the diagnosis and handling of the subjects.

\section{Independent Safety Evaluation Committee}

The Independent Safety Evaluation Committee (ISEC) is responsible for the overall safety of this clinical study. To ensure the safety of the protocol-defined treatment, the ISEC will review the doses and adverse events of 
digoxin, flecainide and sotalol. Specifically, the safety of digoxin will be reviewed when the data on the first three cases are obtained. The safety of flecainide and sotalol will be reviewed when the data on the first case and the first three cases are available. Every serious adverse event will provide the Secretariat within 24 hours of its onset according to the predetermined procedure. The Secretariat then will forward the obtained information without delay to the ISEC for review. The ISEC will notify the investigator of the review results. The serious adverse events will also be reviewed by the ethics committees in the participating institutions including the National Cerebral and Cardiovascular Center according to the Japanese Ethical Guidelines for Clinical Studies and the regulatory notifications regarding the Japanese Advanced Investigational Medical Care Evaluation Program.

\section{Treatment methods}

This study protocol defines all the procedures and schedules that the investigator must abide by to complete this clinical study, including patient selection and registration, transplacental treatment of fetal tachyarrhythmia and follow-up. Registered patients will receive the protocol-defined fetal treatment. As a general rule, the fetal therapy will be administered under 24 hours ECG monitoring in an inpatient setting. The investigator will provide standard pregnancy care, and the pregnant woman will undergo vaginal or caesarean delivery at 37 or more weeks of gestation. If the investigator judges that the pregnancy cannot be maintained to term due to obstetric indications, the investigator will give birth to the fetus according to the Criteria for Delivery (termination of the fetal therapy). If no positive treatment effect is observed in a fetus at 37 gestational weeks, the investigator will consider delivering the fetus without proceeding to further stages of treatment (ie, second or third option or dose increase). If the investigator continues the fetal therapy beyond 37 gestational weeks even when fetal therapy is not effective, the fetus will be classified as non-compliant. The investigator must present a written report that explains the reason for the continuation. Fetuses who completed the fetal therapy will be followed up according to the study schedule. Fetuses in whom the fetal therapy was prematurely discontinued as per the stopping criteria will receive scheduled examinations and other assessments to the extent possible. The stopping criteria define three major conditions: serious adverse drug reactions, death and withdrawal of consent to study participation. If the fetal therapy is prematurely discontinued due to a serious adverse drug reaction to the antiarrhythmic drug, scheduled subsequent examinations and other assessments should be continued to the extent possible. If either the mother or fetus dies during the study period (fetal death, maternal death or postnatal death), the study treatment will be discontinued at this time point. If the mother withdraws her consent to study participation, she and her fetus will be removed from the study. If a registered mother or her fetus is found to have been non-conformant to the eligibility criteria, the mother or fetus will be categorised as non-compliant.

\section{Fetal treatment protocol}

The investigator will provide the fetal therapy as described below. If the investigator finds a positive effect (ie, disappearance of, or improvement in, fetal tachyarrhythmia, see the efficacy endpoints for reference), the investigator will continue the treatment until delivery. If the mother or fetus develops adverse drug reactions to digoxin, sotalol or flecainide, the investigator will either discontinue the drug or reduce its dose at his or her discretion. Overview of the fetal treatment protocol is shown in figure 2.

\section{Fetuses with short VA SVT or AFL}

If the fetus has non-hydrops, the fetal therapy will start with the first-choice therapy. If the fetus is unresponsive to the first-choice therapy, the treatment will proceed to the next step. In cases with fetal hydrops, the fetal therapy will start with the second-choice therapy.

\section{First-choice therapy: digoxin (injections or tablets)}

Rapid saturation is initially performed by $0.5 \mathrm{mg}$ intravenous injection. A $0.25 \mathrm{mg}$ intravenous injection will be administered 8 and 16 hours after the initial dose. If intravenous injections are not feasible, oral administration is performed by $1.5 \mathrm{mg} /$ day in three divided doses. Maintenance oral administration is subsequently performed by $0.75 \mathrm{mg}$ /day in three divided doses. The target blood concentration range is $1.5-2.0 \mathrm{ng} / \mathrm{mL}$. The maintenance doses may be adjusted based on blood concentration measurements. The therapy will be judged ineffective if the fetal arrhythmia does not disappear or improve 3 or more days after the establishment of the target blood level. The investigator will move to the second-choice therapy.

\section{Second-choice therapy: combination of digoxin (injections or} tablets) and sotalol (tablets)

Digoxin is administered or maintained same as the first-choice therapy. Sotalol is administered $160 \mathrm{mg} /$

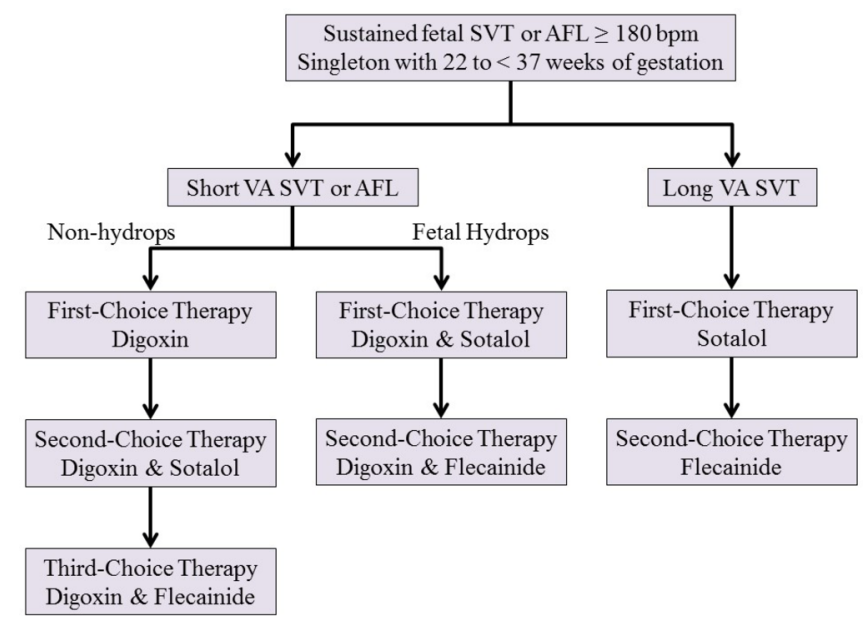

Figure 2 Overview of the fetal treatment protocol. AFL, atrial flutter; SVT, supraventricular tachycardia; VA, ventriculoatrial. 
day in two divided doses as combination therapy. If no improvement is seen with $160 \mathrm{mg}$ /day within 3 days of treatment, proceed to $240 \mathrm{mg}$ / day in two divided doses. If no improvement is seen with $240 \mathrm{mg}$ /day within 3 days of treatment, proceed to $320 \mathrm{mg} /$ day in two divided doses. Sotalol must be discontinued if prolongation of the QT interval is noted (corrected QT interval $>0.5 \mathrm{~s}$ ). The therapy will be judged ineffective if the fetal arrhythmia does not disappear or improve 3 or more days after the start of the highest sotalol dose. The investigator will move to the third-choice therapy.

\section{Third-choice therapy: combination of digoxin (injections or tablets)} and flecainide (tablets)

Digoxin is maintained same as the first-choice therapy. When starting flecainide, the investigator will continue digoxin but discontinue sotalol. Flecainide is administered $200 \mathrm{mg}$ /day in two divided doses as combination therapy. If no improvement is seen with $200 \mathrm{mg} /$ day within 3 days of treatment, proceed to $300 \mathrm{mg} /$ day in two divided doses. Flecainide must be discontinued if prolongation of the QT interval is noted (corrected QT interval $>0.5 \mathrm{~s}$ ). The therapy will be judged ineffective if the fetal tachyarrhythmia does not disappear or improve 3 or more days after the start of the highest flecainide dose.

\section{Fetuses with long VA SVT}

The first-choice therapy is sotalol, regardless of the presence or absence of fetal hydrops. When switching to flecainide, the investigator will discontinue sotalol. The dosage and administration requirements are the same as those for fetuses with short VA SVT.

1. first-choice therapy: sotalol (tablets)

2. second-choice therapy: flecainide (tablets)

\section{Fetuses unresponsive to therapy}

The fetus is defined as unresponsive to the fetal therapy if the fetal tachyarrhythmia does not disappear or improve after treatment with all available options, or the risk of fetal death is strongly suspected due to aggravation of fetal hydrops. The risk of fetal death is strongly suspected if: (1) fetal hydrops continues for 2 or more weeks, or the fetal heart rate is $\geq 180 \mathrm{bpm}$ in the absence of signs of improvement in subcutaneous oedema; (2) fetal hydrops continues for 1 or more weeks, or the fetal heart rate is $\geq 220 \mathrm{bpm}$ in the absence of signs of improvement in subcutaneous oedema; (3) the fetal heart rate monitoring depicts worsening conditions. If the fetus is judged unresponsive to the fetal therapy, the investigator must discontinue the protocol-defined treatment. At his or her discretion, the investigator will decide to deliver the fetus for neonatal treatment or to start other fetal pharmacotherapies. Preferably, the investigator should deliver the fetus by caesarean section, if worsening fetal hydrops strongly suggests progression to fetal death. The investigator should continue collecting data on unresponsive patients.

\section{Stopping criteria}

The investigator must discontinue the protocol-defined treatment when certain events prevent continuation of the protocol treatment, which include the following: (1) a serious adverse drug reaction to the antiarrhythmic drug has developed during the fetal therapy; (2) the investigator's decision to prioritise other therapies than the fetal therapy. When the fetus's parents have withdrawn their consent to study participation, the investigator must discontinue the protocol-defined treatment.

\section{Criteria for delivery}

In responded cases, the investigator should deliver the fetus if: (1) onset of labour; (2) maternal indications suggest that continued pregnancy will cause adverse health effects to the mother; (3) fetal indications suggest an urgent need for delivery. The standard obstetric indications for caesarean section are applicable in this study. The investigator will choose the safest method for the mother and child. Caesarean section is recommended if the investigator cannot monitor the fetal heart rate.

In non-responded cases, the investigator should deliver the fetuses following the previously described criteria in 'fetuses unresponsive to therapy' and 'stopping criteria'.

\section{Follow-up}

The investigator will evaluate the growth and development of the infant subjects. For the follow-up evaluation of the growth and development of the infant subjects, the investigator may ask for the help of developmental paediatricians at own or other medical centres that participate in the Japanese Neonatologist Association. At 18 and 36 months of age, growth and development are assessed. Growth parameters include height, weight, head and chest circumferences. The standard growth charts will be used for reference. The proportion of infants who walk alone and have a vocabulary of three or more words will be determined. The Kyoto Scale of Psychological Development may preferably be used for developmental assessment. The Kyoto scale, standardised neurodevelopment assessment method in Japan, is reported to be well correlated to Bayley III scale for the neurodevelopment follow-up in the cases with very low birth infant. The motor, cognitive and language scores, which are representing specific areas in child development, correlated well between the two tests. ${ }^{18}$

\section{Statistical analysis}

Analyses are done in all fetuses' patients recruited into the study as a primary analysis population, and then per protocol set (ie, removing patients who do not meet the inclusion and exclusion criteria) as a secondary analysis population for sensitivity analysis. All outcome measures are presented as summaries of descriptive statistics (mean (SD) or median (minimum and maximum) for continuous measures, and the numbers and proportions for ordinal and dichotomous measures). For the primary endpoint, that is, the disappearance of fetal 
tachyarrhythmia, an exact CI for the proportion is calculated using Clopper-Pearson method. All analyses are performed according to a prespecified statistical analysis plan using SAS V.9.3 or later (SAS Institute). The Data Coordinating Center at the National Cerebral and Cardiovascular Research Center supports the data management, and statistical analysis and reporting of the study.

\section{Participating institutions}

Case registration requires the approval of the Ethics Committee and Advanced Medical Technology Center. The following institutions will participate in this clinical trial: National Cerebral and Cardiovascular Center, Kurume University School of Medicine, Osaka Medical Center and Research Institute for Maternal and Child Health, National Center for Child Health and Development, Kanagawa Children's Medical Center, Tsukuba University, Toho University Omori Medical Center, Hokkaido University, Hyogo Prefectural Children's Hospital, Nagano Prefectural Children's Hospital, Shizuoka Prefectural Children's Hospital, Kobe City Medical Center Hospital, Mie University, Okayama Medical Center and Osaka University.

\section{DISCUSSION}

To the best of our knowledge, this is the first multicentre clinical trial designed to evaluate the efficacy and safety of the protocol-defined transplacental treatment of fetal tachyarrhythmias.

Prior to designing this clinical trial, we conducted a nationwide survey in Japan from 2004 to 2006 for the purpose of grasping the status of fetal tachyarrhythmia therapy. This study was the first of its kind conducted in Japan. A total of 82 cases were analysed. Forty-one cases $(50 \%)$ were treated in utero. This survey showed that fetal tachyarrhythmia therapy is fairly common, reporting improvements in tachycardia in $37(90.2 \%)$ of 41 cases. Improvements were also reported in $9(81.8 \%)$ of 11 cases of fetal hydrops. These results were comparable with previous reports. ${ }^{3-8}$ One death each was documented in treated and untreated cases. The small number of deaths is probably attributable to proactive interventions informed by previous studies, in which high-risk fetuses had been treated early before the onset of fetal hydrops or underwent preterm delivery. These data indicated that untreated fetuses with tachyarrhythmia would have a much lower mortality rate than that reported in the literature under the natural course of the disease, suggesting that improvement in mortality would not be an adequate endpoint of this clinical study. Our survey also showed that the treated cases were associated with low proportions of preterm delivery, caesarean section and neonatal tachyarrhythmia. These data suggested that fetal therapy improves perinatal maternal and fetal safety.

Based on the above, we have designed this clinical study to establish evidence for fetal tachyarrhythmia therapy. With the participation of many major medical centres providing fetal therapy, this study will evaluate the safety and effectiveness of the protocol developed by the Fetal Arrhythmia Group of the Research Team for EvidenceBased Fetal Therapy (principal investigator: Dr Sago), which was funded under the Health Labour Science Research Grant Program in Japan. The retrospective survey mentioned earlier presents current clinical results of fetal therapy in Japan, which are expected to serve as the control for this prospective study. These data and historical control data from the literature were referenced in designing this clinical study. Therefore, we planned a multicentre single-arm interventional study to confirm the efficacy and safety of the protocol-defined transplacental treatment of fetal tachyarrhythmias.

Our retrospective survey showed that improvements in tachyarrhythmia led to improvements in fetal hydrops, pregnancy maintenance and preterm birth prevention (preterm delivery rate: $12.2 \%$ in treated group vs $41.5 \%$ in untreated group), a lower frequency of caesarean section $(29.7 \%$ in treated group vs $70.7 \%$ in untreated group) and a lower frequency of fetal arrhythmia (48.8\% in treated group vs $78.0 \%$ in untreated group). These have been included as secondary efficacy endpoints. Moreover, this study has been designed to investigate long-term infant growth and development (up to 36 months of age), which are seldom reported in fetal therapy follow-up. ${ }^{19} 20$

\section{Author affiliations}

${ }^{1}$ Department of Perinatology and Gynecology, National Cerebral and Cardiovascular Center (NCVC), Suita, Japan

${ }^{2}$ Department of Pediatrics and Child Health, Kurume University School of Medicine, Kurume, Japan

${ }^{3}$ Center of Maternal-Fetal, Neonatal and Reproductive Medicine, National Center for Child Health and Development, Tokyo, Japan

${ }^{4}$ Department of Pediatric Cardiology, Osaka Medical Center and Research Institute for Maternal and Child Health, Izumi, Japan

${ }^{5}$ Department of Cardiology, Nagano Children's Hospital, Azumino, Japan ${ }^{6}$ Department of Neonatology, Kanagawa Children's Medical Center, Yokohama, Japan

${ }^{7}$ Department of Pediatrics, University of Tsukuba, Tsukuba, Japan

${ }^{8}$ Department of Neonatology, Toho University Omori Medical Center, Tokyo, Japan

${ }^{9}$ Department of Pediatric Cardiology, Saitama Medical University International Medical Center, Hidaka, Japan

${ }^{10}$ Department of Obstetrics and Gynecology, Chiba University, Chiba, Japan ${ }^{11}$ Department of Cardiology, Shizuoka Children's Hospital, Shizuoka, Japan

${ }^{12}$ Department of Internal Medicine, Kanagawa Children's Medical Center, Yokohama, Japan

${ }^{13}$ Department of Pediatric Cardiology, National Center for Child Health and Development, Tokyo, Japan

${ }^{14}$ Department of Cardiovascular Medicine, Nippon Medical School, Tokyo, Japan

${ }^{15}$ Department of Pediatric Cardiology, NCVC, Suita, Japan

${ }^{16}$ Department of Obstetrics and Gynecology, Mie University, Tsu, Japan

${ }^{17}$ Center for Advancing Clinical and Translational Sciences, NCVC, Suita, Japan

${ }^{18}$ Department of Data Science, NCVC, Suita, Japan

Acknowledgements All authors thanked Dr Makoto Nishibatake (chairperson), Dr Masao Nakagawa, Dr Ichiro Kawabata and Dr Naokata Sumitomo for their contribution as the members of the Independent Safety Evaluation Committee in this trial.

Contributors TM, YM, HS, NI, SY, MK, HH, HY, MT, MS, MN, AH, HK, WS, IS, HS, $\mathrm{KU}, \mathrm{SK}, \mathrm{TI}, \mathrm{HY}$ and TH: conception of the study. TM: writing of the manuscript. $\mathrm{KU}$ and HY: coordination of the study. TH: statistical analysis and providing the biostatistical study design. SY, WS, YM and TI: members of the Protocol Evaluation 
Committee. YM and TI: corresponding to this trial. HS: supervision and the grant holder.

Funding This work was supported by a grant from the Ministry of Health, Labor and Welfare of Japan (Health and Labour Science Research Grants for Clinical Research for New Medicine) (H19-009, H23-004), and in part by a grant from the Takeda Science Foundation (J042) and Tsuchiya Foundation (J151).

Competing interests None declared.

Patient consent Obtained.

Ethics approval The Institutional Review Board of the National Cerebral and Cardiovascular Center of Japan.

Provenance and peer review Not commissioned; externally peer reviewed.

Open Access This is an Open Access article distributed in accordance with the Creative Commons Attribution Non Commercial (CC BY-NC 4.0) license, which permits others to distribute, remix, adapt, build upon this work non-commercially, and license their derivative works on different terms, provided the original work is properly cited and the use is non-commercial. See: http://creativecommons.org/ licenses/by-nc/4.0/

(c) Article author(s) (or their employer(s) unless otherwise stated in the text of the article) 2017. All rights reserved. No commercial use is permitted unless otherwise expressly granted.

\section{REFERENCES}

1. Strasburger JF. Prenatal diagnosis of fetal arrhythmias. Clin Perinatol 2005;32:891-912.

2. Strasburger JF, Cheulkar B, Wichman HJ. Perinatal arrhythmias: diagnosis and management. Clin Perinatol 2007;34:627-52.

3. Gembruch U, Yagel S, Fetal Cardiology: Embryology, Genetics, Physiology, Echocardiographic Evaluation, Diagnosis and Perinatal Management of Cardiac Diseases. London, UK: Taylor and Francis, 2005.

4. Vergani P, Mariani E, Ciriello E, et al. Fetal arrhythmias: natural history and management. Ultrasound Med Biol 2005;31:1-6.

5. Krapp M, Kohl T, Simpson JM, et al. Review of diagnosis, treatment, and outcome of fetal atrial flutter compared with supraventricular tachycardia. Heart 2003;89:913-7.
6. Cuneo BF, Strasburger JF. Management strategy for fetal tachycardia. Obstet Gynecol 2000;96:575-81.

7. Oudijk MA, Michon MM, Kleinman CS, et al. Sotalol in the treatment of fetal dysrhythmias. Circulation 2000;101:2721-6.

8. Ebenroth ES, Cordes TM, Darragh RK. Second-line treatment of fetal supraventricular tachycardia using flecainide acetate. Pediatr Cardiol 2001;22:483-7.

9. Jouannic JM, Le Bidois J, Fermont L, et al. Prenatal ultrasound may predict fetal response to therapy in non-hydropic fetuses with supraventricular tachycardia. Fetal Diagn Ther 2002;17:120-3.

10. Oudijk MA, Ruskamp JM, Ververs FF, et al. Treatment of fetal tachycardia with sotalol: transplacental pharmacokinetics and pharmacodynamics. J Am Coll Cardiol 2003;42:765-70.

11. Fouron JC, Fournier A, Proulx F, et al. Management of fetal tachyarrhythmia based on superior vena cava/aorta Doppler flow recordings. Heart 2003;89:1211-6.

12. Jaeggi ET, Carvalho JS, De Groot E, et al. Comparison of transplacental treatment of fetal supraventricular tachyarrhythmias with digoxin, flecainide, and sotalol: results of a nonrandomized multicenter study. Circulation 2011;124:1747-54.

13. Krapp M, Baschat AA, Gembruch U, et al. Flecainide in the intrauterine treatment of fetal supraventricular tachycardia. Ultrasound Obstet Gynecol 2002;19:158-64.

14. Strasburger JF, Cuneo BF, Michon MM, et al. Amiodarone therapy for drug-refractory fetal tachycardia. Circulation 2004;109:375-9.

15. D'Alto M, Russo MG, Paladini D, et al. The challenge of fetal dysrhythmias: echocardiographic diagnosis and clinical management. J Cardiovasc Med 2008;9:153-60.

16. Jaeggi ET, Nii M. Fetal brady- and tachyarrhythmias: new and accepted diagnostic and treatment methods. Semin Fetal Neonatal Med 2005;10:504-14.

17. Jaeggi E, Fouron JC, Fournier A, et al. Ventriculo-atrial time interval measured on $\mathrm{M}$ mode echocardiography: a determining element in diagnosis, treatment, and prognosis of fetal supraventricular tachycardia. Heart 1998;79:582-7.

18. Kono Y, Yonemoto N, Kusuda S, et al. Developmental assessment of VLBW infants at 18 months of age: A comparison study between KSPD and Bayley III. Brain Dev 2016;38:377-85.

19. Oudijk MA, Gooskens RH, Stoutenbeek P, et al. Neurological outcome of children who were treated for fetal tachycardia complicated by hydrops. Ultrasound Obstet Gynecol 2004;24:154-8.

20. Lopriore E, Aziz MI, Nagel HT, et al. Long-term neurodevelopmental outcome after fetal arrhythmia. Am J Obstet Gynecol 2009;201:46. e1-46.e5. 